\title{
Characterization of Monocytes-targeted Nanocarriers Biodistribution in Leukocytes in ex-vivo and in-vivo Models
}

\author{
Einat Cohen-Sela ${ }^{1}$, Michael Chorny², Dikla Gutman ${ }^{1}$, Sivan Komemi ${ }^{1}$, Nickolay Koroukhov ${ }^{1}$, Gershon Golomb ${ }^{*}, 1$ \\ ${ }^{1}$ Institute for Drug Research, School of Pharmacy, Faculty of Medicine, The Hebrew University of Jerusalem. ${ }^{2}$ Cardiology Research, \\ The Children's Hospital of Philadelphia, Philadelphia, PA 19104 \\ Corresponding author: gershong@ekmd.huji.ac.il
}

\begin{abstract}
Aims: On intravenous administration, unprotected nanocarriers are rapidly captured by the mononuclear phagocytic system (MPS). This has been exploited for passive targeting of nanoparticulate drug delivery systems for depleting circulating monocytes and macrophages for the treatment of various disorders. However, the extent of nanocarriers biodistribution among leukocytes, has not been characterized. The aim of this work was to examine the biodistribution of monocyte-targeted nanoparticles and liposomes in leukocytes. Materials \& Methods: Fluorescently-labeled large negative liposomes (LN-LIP, $130 \pm 33 \mathrm{~nm},-25.2 \pm 6.3 \mathrm{mv}$ ), large negative NP (LN-NP, $133 \pm 40 \mathrm{~nm},-40 \pm 8 \mathrm{mv}$ ), large positive NP (LP-NP, $121 \pm 36 \mathrm{~nm},+62 \pm 9 \mathrm{mv}$ ), and small negative NP (SN-NP, $85 \pm 26 \mathrm{~nm},-$ $15 \pm 2 \mathrm{mv}$ ) were formulated. Fluorescence activated cell sorting (FACS) was utilized to determine internalization in rabbit's blood both ex-vivo and in-vivo. Results \& Conclusions: Monocytes, neutrophils, and lymphocytes internalized the NP. SN-NP exhibited the highest selectivity towards monocytes. Granulocytes preferentially internalized LN-LIP in comparison to all polymeric NP formulations. NP charge had no effect on their engulfment by granulocytes, while NP size was found to be an important factor as granulocytes showed preference towards large NP formulations (LN-NP and LP-NP). Lymphocytes preferentially internalized large negatively charged formulations (LN-LIP and -NP). The ex-vivo results failed to predict in-vivo results. Formulations targeted to monocytes distribute into other leukocytes as well, which should be determined in-vivo.
\end{abstract}

Keywords: Liposomes; Nanoparticles (NP); UptakeInternalization; Endocytosis; Monocytes; Leukocytes

Citation: E. Cohen-Sela, et al. Characterization of Monocytes-targeted Nanocarriers Biodistribution in Leukocytes in ex-vivo and in-vivo Models. Nano Biomed. Eng. 2010, 2(2), 91-99. DOI: 10.5101/nbe.v2i2.p91-99.

\section{Introduction}

Nanocarrier systems, such as liposomes and polymeric nanoparticles (NP), have been increasingly employed as drug delivery systems for proteins, macromolecules, small molecules and nucleic acids [1, 2]. The nanocarriers are utilized to protect molecules from biodegradation as well as to modify their pharmacokinetics and biodistribution [3].

Numerous articles regarding the fate of IV injected particles highlight the role of the mononuclear phagocytic system (MPS) as the scavenger of the foreign nanocarriers [4-6]. The interaction of the nanocarriers with opsonins, depending on their physicochemical properties [7], results in their recognition and endocy- tosis. Therefore, several methods of masking the nanocarriers have been developed, in an attempt to temporarily bypass the MPS extending their circulation time [8]. On the other hand, the natural fate of nanocarriers in the MPS was exploited for passive targeting to monocytes and macrophages affecting various disorders including infections, rheumatoid arthritis and restenosis [9-13]. Depletion of macrophages and circulating monocytes in-vivo has been achieved by particulate delivery systems containing bisphosphonates (BP). The nanocarriers including liposomes, polymeric NP and albumin NP enable intracellular delivery of the BP which inactivate and kill these cells [11, 12, 14-18], alleviating the inflammatory state. Thus, inhibition of 
restenosis and endometriosis has been demonstrated following a single IV injection of liposomes containing alendronate [11, 16, 17, 19]. Polymeric NP containing alendronate have been also examined in restenosis therapy $[15,20]$. However, the extent of both liposomes and NP containing BP internalization by leukocytes other than monocytes has not been characterized. Moreover, the effect of NP's charge and size on their biodistribution among monocytes, granulocytes (neutrophils) and lymphocytes has not been determined. Characterization of the biodistribution of nanocarriers and understanding the governing factors is important for designing a more selective formulation. Selectivity of such particulate dosage forms could result in enhanced therapeutic action while minimizing nonspecific distribution and possible side effects.

In this work the biodistribution of NP and liposomes in leukocytes has been examined. In order to explore the effect of nanocarriers' type and physicochemical properties on the extent of internalization by leukocytes, fluorescent, negatively and positively charged polymeric NP, of various size, have been formulated. The uptake of these formulations by macrophages in-vitro, and by monocytes, granulocytes and lymphocytes in rabbit's blood both ex-vivo and in-vivo was studied in comparison to the standard negatively charged liposomes [9].

\section{Materials and Methods \\ 2.1 NP preparation}

NP were prepared by the nanoprecipitation technique [21]. Briefly, $300 \mathrm{mg}$ polymer composed of $75 \%$ poly (D,L-lactide-co-glycolide) 50:50 (PLGA, SigmaAldrich, Rehovot, Israel) and $25 \%$ poly (DL-lactide) (PLA) covalently labeled with BODIPY FL 505/515 (a gift from Dr. Ivan S. Alferiev, University of Pennsylvania, Philadelphia, USA [22]) were dissolved in $30 \mathrm{ml}$ acetone (JT Baker, Deventer, The Netherlands). The organic phase $(30 \mathrm{~mL})$ was injected into a $150 \mathrm{~mL}$ aqueous phase containing $60 \mathrm{mg}$ of pluronic F68 (Sigma-Aldrich, Rehovot, Israel) or cetyltrimethylammonium bromide, (CTAB, Sigma-Aldrich, Rehovot, Israel) to formulate large negatively charged NP (LN-NP) and large positively charged NP (LP-NP), respectively. NP were formed spontaneously and the organic solvents and excess of water were evaporated under reduced pressure by means of a rotary evaporator (Buchi, Flawil, Switzerland). Small negatively charged NP (SNNP) were prepared similarly with the inclusion of 30 $\mathrm{ml}$ ethanol (JT Baker, Deventer, The Netherlands) in the organic phase and the use of a $600 \mathrm{~mL}$ aqueous phase containing $300 \mathrm{mg}$ pluronic $\mathrm{F} 68 . \mathrm{NaCl}$ was dissolved in water and was added to the NP formulations to achieve isotonicity. The NP were purified using a sephadex G50 column and were filtered through 0.45 $\mu \mathrm{m}$ hydrophilic syringe filter (Sartorius, Goettingen, Germany) to remove residual aggregates.

\subsection{Liposome preparation}

The biodistribution of polymeric NP in leukocytes was studied in comparison to liposomes. Large negative liposomes (LN-LIP) were formulated by a modified thin lipid film hydration method [23] as was described previously [9].

\subsection{Endocytosis in-vitro \\ 2.3.1 Macrophages cell culture}

Unless otherwise noted all materials for cell cultures were purchased from Biologic Industries (Beit Haemek, Israel) and from Sigma. A murine macrophage cell line (RAW 264, ATCC, Rockville, MD, USA) was utilized. Cells were grown in DMEM supplemented with $10 \%$ fetal calf serum, $2 \mathrm{mM}$ L-glutamine, 100 units $/ \mathrm{ml}$ penicillin and $100 \mu \mathrm{g} / \mathrm{ml}$ streptomycin in $5 \% \mathrm{CO}_{2}$ atmosphere at $37^{\circ} \mathrm{C}$. The cells were plated at $5 \times 10^{5}$ cells per well on 12-well plates and allowed to grow overnight. Uptake of the NP and LN-LIP by RAW 264 was determined by incubating the cells with the nanocarriers for $1 \mathrm{hr}$ or $4 \mathrm{hr}$. After incubation, cells were washed twice with PBS, detached from the plate using trypsin and collected into a test tube. Cells were washed (X1500 RPM, 5 min) in FACS medium (PBS, $1 \%$ BSA, $0.02 \%$ sodium azide) and suspended in $0.75 \mathrm{~mL}$ FACS medium for flow cytometry. The number of cells that engulfed NP was determined in comparison to untreated cells.

\subsubsection{Human and rabbit's blood ex-vivo}

Blood from healthy human donors or rabbits was incubated with NP or LN-LIP. Incubation was performed in test tubes containing EDTA as an anticoagulant (BD, NJ, USA) for $1 \mathrm{hr}$ and $4 \mathrm{hr}$ at $37^{\circ} \mathrm{C}$. Blood samples were analyzed by FACS as previously described [11]. In short, Rabbit's blood $(100 \mu \mathrm{l})$ was incubated for 30 $\min \left(4{ }^{\circ} \mathrm{C}\right.$, in the dark) with mouse anti-human RPEconjugated anti-CD14 (DAKO, Cambridge, UK). Human blood was incubated for $30 \mathrm{~min}\left(4^{\circ} \mathrm{C}\right.$, in the dark) with mouse anti-human RPE-conjugated anti-CD14 and/or anti CD3. FACS lysing solution (1:10 dilution) was added for $10 \mathrm{~min}$. The residual cells were washed (X1500 RPM, 5 min) in FACS medium (PBS, $1 \%$ BSA, $0.02 \%$ sodium azide) and suspended in $1 \mathrm{~mL}$ FACS medium for flow cytometry. Monocytes, granulocytes and lymphocytes were identified according to their relative size, side-scattering, and fluorescence. Untreated blood samples were labeled with the antibody to determine the background levels of nanocarriers' fluorescence. Uptake was expressed as the extent of cells containing fluorescence above baseline levels.

\subsection{Endocytosis in-vivo}

New Zealand white rabbits (Harlan Laboratories), weighing $2.5-3.5 \mathrm{~kg}$ were randomly assigned to treatment groups. Blood was extracted before, and $1 \mathrm{hr}, 4 \mathrm{hr}$ and $24 \mathrm{hr}$ after IV injection of LN-LIP ( $\mathrm{n}=4)$, LN-NP 
$(n=4)$, LP-NP $(n=3)$ and SN-NP $(n=3)$. Blood samples were analyzed by FACS as described above for ex-vivo samples (3.2).

\subsection{Statistics}

Results are expressed as the mean \pm SD. Statistical difference between groups was tested using one-tailed unpaired Student's t test, and a 2-way ANOVA analysis followed by Tukey-test for multiple comparisons. Differences were termed statistically significant at $\mathrm{p}<0.05$.

\section{Results}

\subsection{Formulations}

The properties of the liposomal and NP formulations used in order to examine the effect of nanocarriers charge and size on the distribution in cells are summarized in Table 1. The LN-LIP formulation utilized is similar to that reported in studying the effect of liposomal bisphosphonates on restenosis [11, 16, 17, 24] and was composed of DSPC, DSPG and cholesterol with a size of $130 \pm 33 \mathrm{~nm}$ and a negative zeta potential $(-25.2 \pm 6.3 \mathrm{mv})$. The LN-NP formulation was composed of PLGA and had comparable size and charge to LN-LIP, $133 \pm 40 \mathrm{~nm}$ and $-40 \pm 8 \mathrm{mv}$. The LP-NP were $121 \pm 36 \mathrm{~nm}$ size and with a zeta potential of $+62 \pm 9 \mathrm{mv}$, and the SN-NP size was $85 \pm 26 \mathrm{~nm}$ with a charge of $15 \pm 2 \mathrm{mv}$.

\subsection{Endocytosis by macrophages in-vitro}

In a preliminary experiment we verified that macrophages internalize the formulations. The nanocarriers were incubated with Raw 264 macrophages and sam- ples were analyzed by FACS. Fig. 1 demonstrates the number of Raw 264 cells that have engulfed NP after 1 $\mathrm{hr}$ and $4 \mathrm{hr}$ of incubation. It can be seen that for all polymeric NP formulations examined were engulfed by the entire macrophage population, above $96 \%$ after 1 $\mathrm{hr}$ and a similar percentage after $4 \mathrm{hr}$. In contrast, the rate of LN-LIP engulfment was significantly lower after $1 \mathrm{hr}, 86 \%$, and was increased to $93 \%$ after $4 \mathrm{hr}$.

Table 1 Size and zeta potential of fluorescent liposomes (Hydroxypyrene trisulfonic acid, HPTA in the hydrophilic core) and of fluorescent NP formulations (PLA covalently bound with bodipy)

\begin{tabular}{lccc}
\hline Nanocarrier & Size $(\mathbf{n m})$ & PDI & Zeta $(\mathbf{m v})$ \\
\hline Liposomes & $130 \pm 32.7$ & 0.065 & $-25.2 \pm 6.3$ \\
Large negative NP(LN-NP) & $133.3 \pm 40.1$ & 0.091 & $-39.9 \pm 7.5$ \\
Large positive NP (LP-NP) & $121.1 \pm 35.9$ & 0.088 & $+62.2 \pm 9.3$ \\
Small negative NP(SN-NP) & $84.8 \pm 25.6$ & 0.091 & $-14.6 \pm 1.7$ \\
\hline
\end{tabular}

\subsection{Formulation effects on the biodistribution in} leukocytes

\subsubsection{Identifying the cells internalizing NP}

In a preliminary experiment we have identified the cells participating in the uptake of the formulations. Labeling with anti CD14 antibody enabled identification of three cells population, monocytes, granulocytes and lymphocytes, which internalize LN-NP in human blood (Fig. 2a) and rabbit's blood (Fig. 2f). All of the monocytes and some of the granulocytes and of the lymphocytes have endocytosed LN-NP (Fig. 2c and 2h).

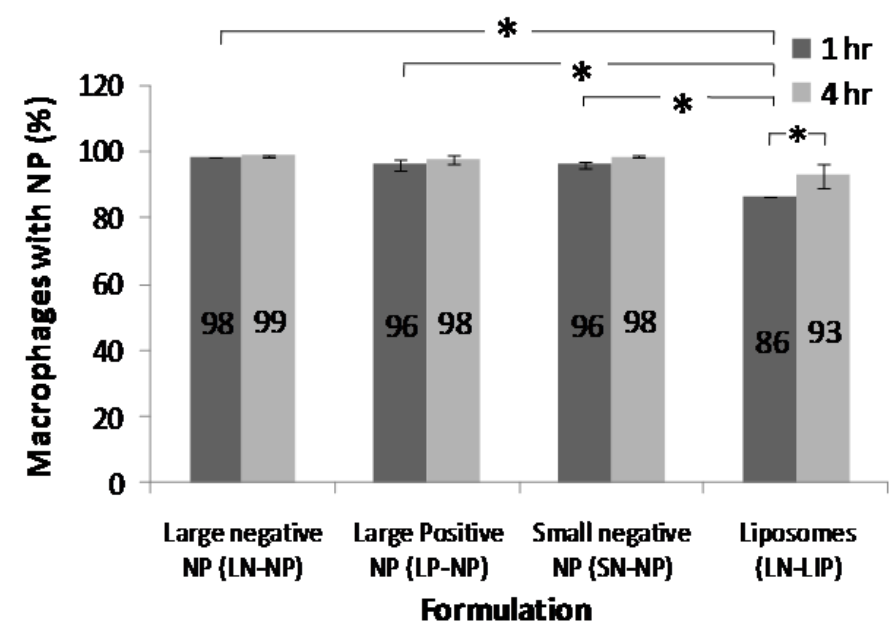

Figure 1. The effect of formulation type, size and surface charge on liposomes and polymeric NP internalization by RAW 264 macrophages following incubation in-vitro. Uptake was quantified by means of fluorescent activated cell sorting (FACS) of liposomes labeled with hydroxypyrene trisulfonic acid (HPTA) in the hydrophilic core and polymeric NP covalently bound with bodipy. 
In order to examine which lymphocytes endocytose NP, human blood was incubated with fluorescent NP, and specimens were double labeled with the antibody against CD14 (FL2 fluorescence, a marker for monocytes) and an antibody against CD3 (FL3 fluorescence), which is a marker for $\mathrm{T}$ cells. The T-cells population was found as the only one positive for CD3 fluorescence (above the horizontal line, Fig. $2 \mathrm{~d}$ and e). Since no overlapping between the fluorescence of $\mathrm{NP}$ and CD3 was observed (upper right quadrant, Fig. 2e), it can be concluded that the $\mathrm{T}$ cells did not endocytose NP. Since no antibodies for rabbit's B and T cells were available, these cells could not be distinguished in rabbits blood. However, based on the human blood experiments it is suggested that $\mathrm{B}$ cells were the ones to engulf the NP.

\subsubsection{The biodistribution in rabbit's blood}

In order to examine the effect of size and charge on leukocytes uptake both ex-vivo and in-vivo, rabbits were chosen as the animal model.

\subsubsection{Endocytosis ex-vivo}

In order to examine the uptake of NP and LN-LIP by leukocytes ex-vivo, rabbit's blood was incubated with the formulations for $1 \mathrm{hr}$ and $4 \mathrm{hr}$, labeled with anti CD14 antibody and analyzed by FACS. After $1 \mathrm{hr}$ of incubation negatively charged NP were endocytosed by monocytes at a larger extent $(92.7 \%$ and $87.9 \%$, LNNP and SN-NP, respectively, Fig. 3a, Table 2, Tables 2-7 can be seen the supplementary files) in comparison to LP-NP and LN-LIP (53.1 \% and $36.4 \%$, respectively, Fig. 3a, Table 2). After $4 \mathrm{hr}$ of incubation, increased uptake rate was observed for all formulations; the negatively charged NP showed higher uptake rates with $99.3 \%$ for both LN-NP and SN- NP (Fig. 3a, Table 2). LP-NP exhibited the lowest uptake values of $62.9 \%$, and the LN-LIP were markedly affected by incubation time with more than a twofold increase $(86.7 \%$, Fig. 3a, Table 2). Granulocytes (Fig. 3b, Table 3, see the supplementary files) were also found to preferably engulf LN-NP after $1 \mathrm{hr}$, with uptake of $77.3 \%, 56.6 \%$ and $64.4 \%$ for LN-NP, LP-NP and SN-NP, respectively. In addition, the uptake extents by granulocytes for all NP formulations were found to be significantly higher than LN-LIP (15.1 \%, Fig. 3b, Table 3). After 4 hr, the uptake of both LN-NP and SN-NP by granulocytes was found to be favored in comparison to LP-NP and LN-LIP (Fig. 3b, Table 3). LP-NP engulfment by granulocytes was not affected by incubation time and their extent of endocytosis remained unchanged (52.9\%), while the extent LN- NP and SN- NP uptake was increased to $87.5 \%$ and $80.8 \%$, respectively (Fig. 3b, Table 3). LN-LIP uptake by granulocytes was increased three fold after $4 \mathrm{hr}$ of incubation (to $46.1 \%$, 3b, Table 3).
Both LN-NP and LP-NP were found to be preferably engulfed by lymphocytes ex-vivo after $1 \mathrm{hr}$.
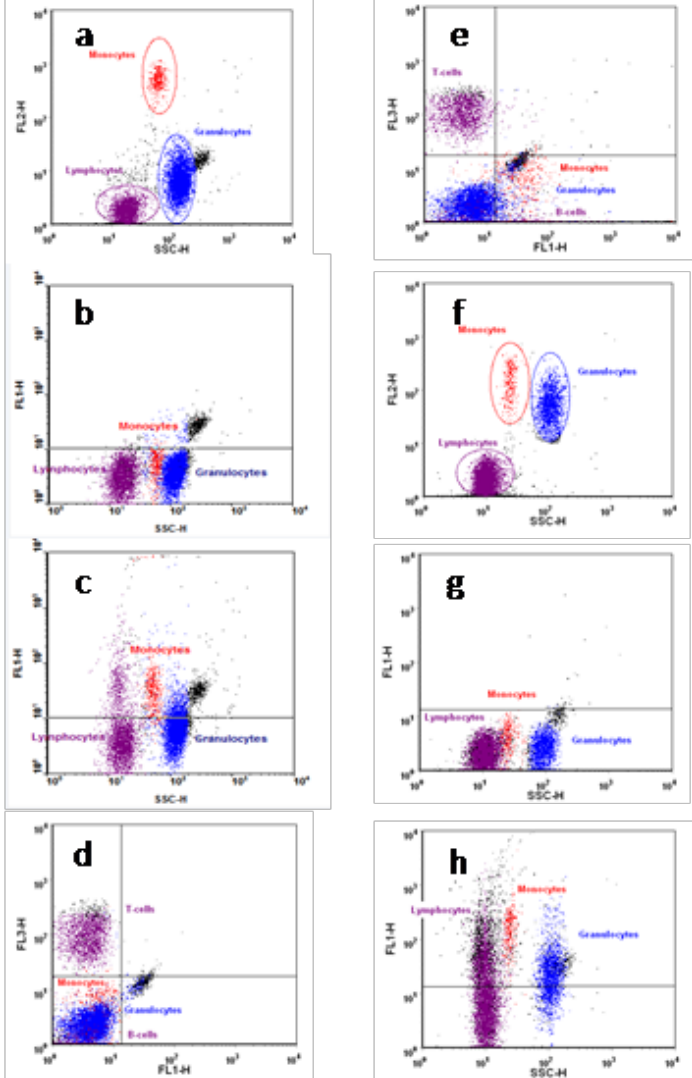

Figure 2. In order to identify the cells that engulf nanoparticles (NP), fluorescent large negative polymeric NP (LN-NP, $133.3 \mathrm{~nm},-39.9 \mathrm{mV}$ ) were incubated with whole anticoagulated human and rabbit's blood for 60 min. NP were labeled by bodipy covalently bound to PLA. FL1, FL2 and FL3 indicate NP, CD14 and CD3 fluorescence intensity, respectively. Representative fluorescence activated cell sorting (FACS) analysis demonstrating anti CD14 labeling of human non treated peripheral blood ( $\mathrm{a}$ and $\mathrm{b}$ ) and following incubation (c).CD14 and anti CD3 labeling of human non treated peripheral blood (d), and following incubation (e). Anti CD14+ labeling of rabbits non treated peripheral blood (f and g) and following incubation (h).

Three cell types can be distinguished in human (a) and rabbit's blood (f), CD14 positive cells (monocytes, red, granulocytes, blue) and CD14 negative cells (lymphocytes, purple).

The horizontal line in b,c, g and $\mathrm{h}$ represents the baseline above which cells were considered for analysis. All cells types engulfed the polymeric NP and were found positive for NP fluorescence (c and h).

The vertical line in e represents the baseline above which cells were considered for analysis. No overlap was detected between the NP and CD3 fluorescence (upper right quadrant), indicating that T-cells did not engulf the polymeric NP. 


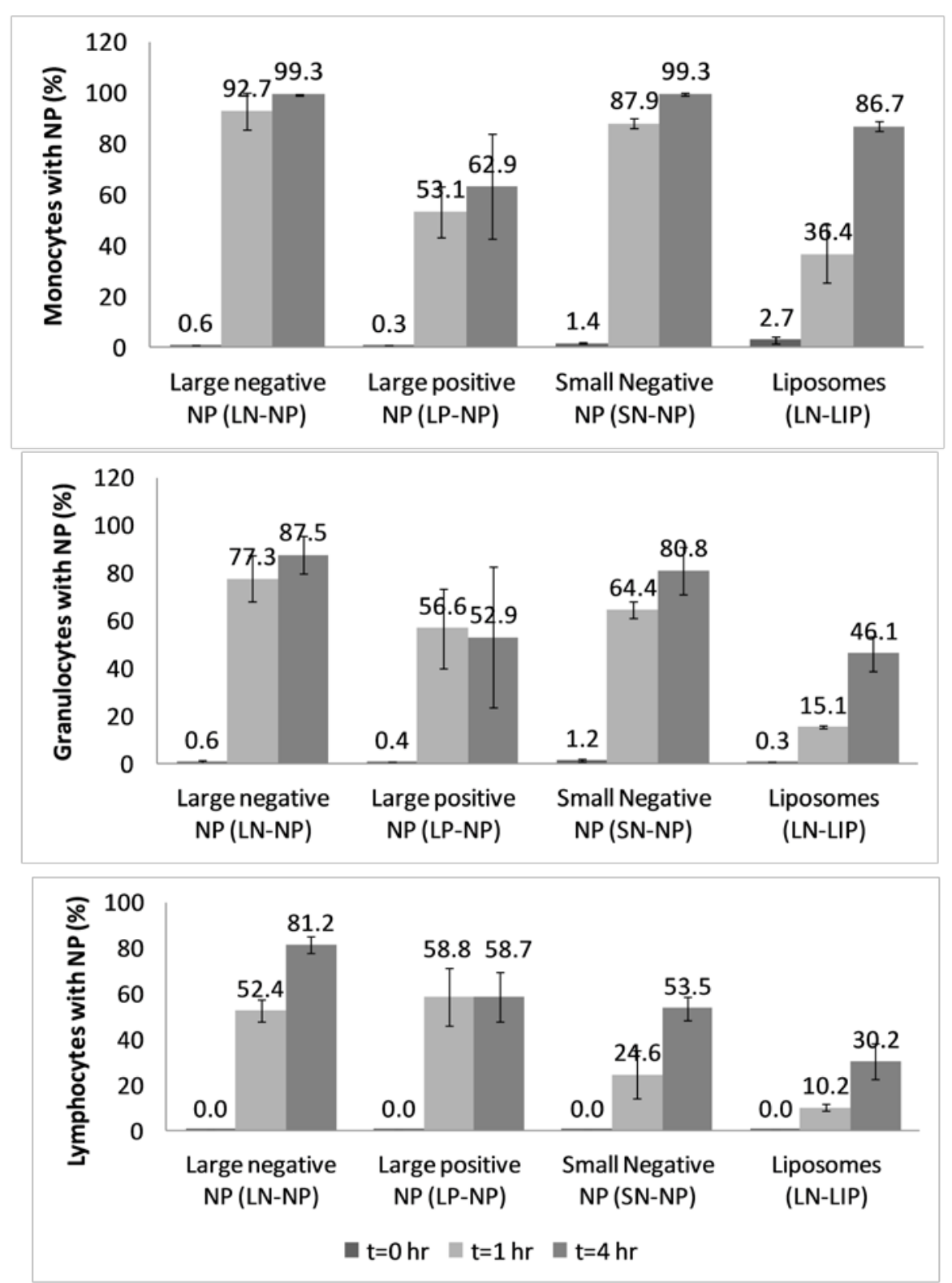

Figure 3. The effect of formulation type, size and surface charge on liposomes and polymeric NP internalization by monocytes (a), granulocytes (b) and lymphocytes (c) in rabbit's peripheral blood following incubation ex-vivo. Uptake was quantified by means of fluorescent activated cell sorting (FACS) of liposomes labeled with Hydroxypyrene trisulfonic acid (HPTA) in the hydrophilic core and polymeric NP covalently bound with bodipy incubation, with an uptake of $52.4 \%$ and $58.8 \%$, respectively (Fig. 3c, Table 4, see the supplementary files), in comparison to $24.6 \%$ and $10.2 \%$, SN-NP and LN-LIP, respectively. After 4hr the uptake of LN-NP was higher in comparison to all other formulations (81.2 \%), while the uptake of LP- NP was not affected by incubation time and remained unchanged (58.7 \%, Fig. 3c, Table 4). The extent of uptake of SN- NP and LN-LIP was increased by two and three fold after $4 \mathrm{hr}$ of incubation, $53.5 \%$ and $30.2 \%$, respectively.

\subsubsection{Endocytosis in-vivo}

In order to examine the in-vivo biodistribution of the various formulations in leukocytes, NP and LN-LIP were injected to rabbits and blood specimens were analyzed immediately before, and $1 \mathrm{hr}, 4 \mathrm{hr}$ and $24 \mathrm{hr}$ after injection. Since no monocytes were detected in the blood stream $1 \mathrm{hr}$ post injection (Fig. 4), in order to obtain the correct extent of monocytes uptake, the disappeared monocytes were considered as NP engulfed monocytes. Uptake extent was calculated by summing the percentage of the disappeared monocytes and of the monocytes containing fluorescent nanocarriers. The whole monocytes population was found to engulf all formulations tested $1 \mathrm{hr}$ after injection, with an uptake extent of over $95 \%$ (Fig. 5a, Table 5, see the supplementary files). A decrease in uptake was observed for all the formulations $4 \mathrm{hr}$ after injection, $77.2 \%$ and $69.8 \%$, LN-NP and LP-NP, respectively. A more pro- 
see the supplementary files). The uptake of LN-LIP

nounced decrease of almost two fold was observed for SN-NP and LN-LIP, $50.5 \%$ and $48.6 \%$, respectively (Fig. 5a, Table 5). The uptake of all formulations by monocytes in-vivo was significantly reduced $24 \mathrm{hr}$ post injection. LN-NP, LP-NP, SN-NP and LN-LIP were endocytosed by $15.4 \%, 11.2 \%, 6.4 \%$ and $4.4 \%$ of the monocytes, respectively (Fig. 5a, Table 5).

Granulocytes preferably engulfed LN-LIP with a significantly larger extent of $71.3 \%$ in comparison to the NP formulations $1 \mathrm{hr}$ after injection (Fig. 5b, Table 6). Large NP, both negatively and positively charged, showed similar uptake extent of $21.0 \%$ and $21.9 \%$, respectively. In contrast, SN-NP were not engulfed by granulocytes in-vivo (2.1\%, Fig. 5b, Table 6). The uptake rate of large NP and LN-LIP was reduced $4 \mathrm{hr}$ after injection, but remained significantly higher for LN-LIP in comparison to LN- NP (47.8\% and $16.3 \%$, respectively) and in comparison to LP-NP (9.5\%). SN-NP were not engulfed by granulocytes in-vivo $4 \mathrm{hr}$ and 24 hr after injection, $2.5 \%$ and $1.3 \%$, respectively (Fig. $5 \mathrm{~b}$, Table 6 , see the supplementary files). The uptake of LN-NP and LP-NP was reduced after $24 \mathrm{hr}, 12.5 \%$ and $2.0 \%$, respectively (Fig. 5b, Table 6). In contrast, LNLIP uptake was increased to $57.1 \% 24 \mathrm{hr}$ after injection, which was found to be insignificantly different than the uptake after $4 \mathrm{hr}$.
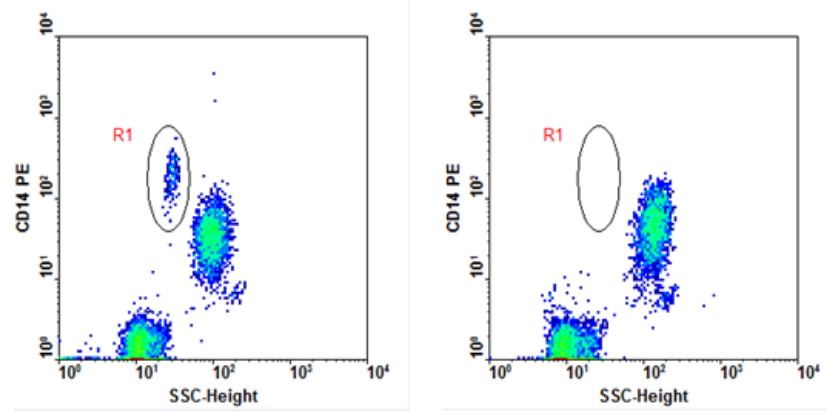

Figure 4. Monocytes population (circled), "disappeared" from the blood stream $1 \mathrm{hr}$ after nanocarrires injection. Representative fluorescence activated cell sorting (FACS) analysis demonstrating CD14+ labeling of rabbits non treated peripheral blood (a); (b) $60 \mathrm{~min}$ after IV injection of large negative fluorescent polymeric NP (133 nm, -39.9 mv, b); SSC indicates sidescattering, FL1 and FL2 indicate NP and antibody fluorescence intensity, respectively.

Lymphocytes uptake (Fig. 5c, Table 7, see the supplementary files) $1 \mathrm{hr}$ after injection was found to be similar to LN-NP (23.6 \%) and LN-LIP (25.3 \%), and significantly higher than the uptake of LP-NP (9.9\%) and SN- NP (4.2\%). The uptake by lymphocytes $4 \mathrm{hr}$ after injection was slightly reduced and remained similar for LN-NP and LN-LIP, $19.6 \%$ and $19.1 \%$, respectively, and was significantly higher in comparison to LP-NP (4.7 \%) and SN-NP (0.7 \%, Fig. 5c, Table 7, after $24 \mathrm{hr}$ remained unchanged (21.05\%) and was significantly higher than the uptake of all other formulations, $4.0 \%, 0.7 \%$ and $0.2 \%$, LN- NP, LP-NP and SN-NP, respectively.

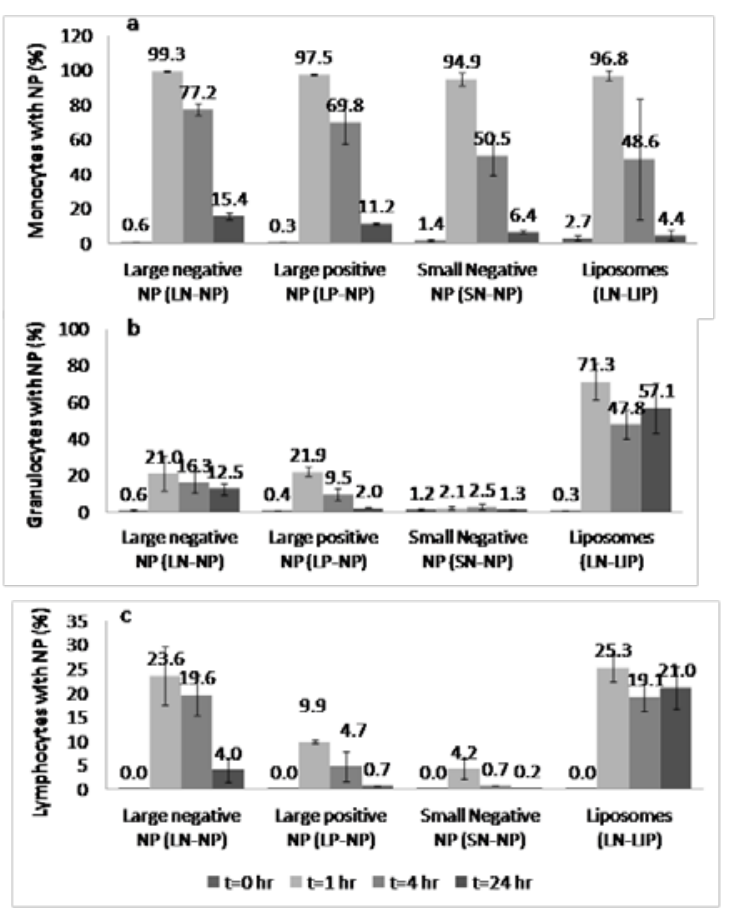

Figure 5. The effect of formulation type, size and surface charge on liposomes and polymeric NP internalization by monocytes (a), granulocytes (b) and lymphocytes (c) in rabbit's peripheral blood $1 \mathrm{hr}, 4 \mathrm{hr}$ and 24 $\mathrm{hr}$ after IV injection (円3). Uptake was quantified by means of fluorescent activated cell sorting (FACS) of liposomes labeled with Hydroxypyrene trisulfonic acid (HPTA) in the hydrophilic core and polymeric NP covalently bound with bodipy.

\section{Discussion}

By encapsulating BP in charged liposomes or polymeric NP, these bone-seeking molecules are targeted to circulating monocytes and macrophages [11, 14-17]. Transient suppression of monocytes/macrophages function by NP-mediated manipulation has been shown to improve the treatment of a variety of disorders involving these cells [9-13]. The aims of this study was to examine the biodistribution of monocytes- targeted polymeric NP and liposomes in all leukocytes, both invitro and in-vivo, as well as determine the effect of formulation characteristics and examine the correlation between the in-vitro and in-vivo models. The results show that formulations targeted to monocytes are also taken up by granulocytes and lymphocytes. The formulations' characteristics were found to affect their biodistribution and the ex-vivo model failed to predict the in-vivo results. 
4.1 Endocytosis of nanocarriers by macrophages invitro

Formulations' uptake by Raw 264 macrophages invitro was examined (Fig. 1) in order to preliminary verify their suitability for monocytes targeting. While polymeric NP were engulfed by the entire population ( $\geq 96 \%$ ) of the macrophages within $1 \mathrm{hr}$ of incubation, LN-LIP uptake was slower and some progress in the engulfment extent between $1 \mathrm{hr}$ and $4 \mathrm{hr}$ was observed. This may be explained by the liposomes lower density. Liposomes have a specific weight similar to that of water [25], hence they do not sediment onto the cells surface in cell culture conditions. In contrast, the NP formulated in this work are nanospheres and their density is about $1.4 \mathrm{~g} / \mathrm{ml}$ [26], which can cause their sedimentation and accelerate their internalization by the cells.

\subsection{Endocytosis of nanocarriers by leukocytes}

Following ex-vivo incubation with human and rabbit's blood, three types of leukocytes engulfing nanocarriers were identified, monocytes, which are the cells of target, granulocytes and lymphocytes (Fig. 2c and h). Granulocytes are polymorphonuclear cells that have been noted in the uptake of NP [9, 27]. It has been shown that neutrophils, which comprise the majority of the granulocytes population, are the phagocytic cells [28]. Although they are scarcely mentioned in the literature as particle capturers, due to the relatively low number of monocytes, the neutrophils represent the main phagocytes in the circulation [28].

The lymphocytes population is composed mainly of B cells, T cells, and about $2 \%$ of the population is natural killer cells (NK). None of the lymphocytes are phagocytes cells by definition. However, B cells are antigen presenting cells that are known to engulf soluble antigens by receptor mediated endocytosis [29]. In addition, it was demonstrated that $\mathrm{B}$ cells are the lymphocytes which endocytose NP in human blood (Fig. 2d and e). Therefore we assumed that B cells are the ones that engulfed NP in rabbit's blood as well. The literature reference to nanocarriers' engulfment by lymphocytes is scarce. Prior works on lymphocytes uptake were limited to certain formulations in-vitro [30-32]. In the present both ex-vivo and in-vivo models have been utilized to investigate the uptake of polymeric NP of various charge and size, and of liposomes by leukocytes. In addition, the correlation between the ex-vivo and in-vivo models was examined. In order to assure that the biodistribution of the NP is observed, a polymer covalently labeled with bodipy was used for NP labeling. LN-LIP were labeled by a highly hydrophilic compound encapsulated in the internal aqueous phase, since it does not leak out of intact liposomes.

\subsection{Correlation between ex-vivo and in-vivo models}

The ex-vivo model failed to predict the uptake of the formulations by blood white cells in-vivo. The uptake of LP-NP by monocytes ex-vivo was found to be significantly lower in comparison to in-vivo. This could be due to the interaction between the positively charged surface of the NP and the negatively charged proteins in the blood that was previously shown to create aggregates [33]. When placed in a tube, the probability of cell-particle encounters and engulfment is higher and may explain the enhanced uptake of polymeric NP by granulocytes and lymphocytes ex-vivo vs. in-vivo. On the other hand, LN-LIP were engulfed to a higher extent in-vivo, probably due to their lower density [25], which reduces their sedimentation on the cells surface ex-vivo as was observed in-vitro.

\subsection{The effect of size and charge on leukocytes up- take in-vivo}

Virtually no monocytes were detected in the blood stream $1 \mathrm{hr}$ following IV injection (Fig. 4), which could be explained that phagocytosis of the nanocarriers triggered monocyte margination as has been shown previously [34]. The uptake extent by monocytes $4 \mathrm{hr}$ after injection decreased for all formulations. This could be attributed to the appearance of new monocytes from the bone marrow [34] or from the monocytes reservoir in the spleen [35], in addition to the margination of nanocarriers engulfing monocytes observed after $1 \mathrm{hr}$.

The size, charge and type of the nanocarriers had no effect on endocytosis by the target cell, the monocytes. All the formulations were engulfed by the entire monocytes population and were found suitable for targeting to these cells. Formulation characteristics affected internalization by granulocytes and lymphocytes (B cells), hence affecting the selectivity of the carriers to monocytes. LN-LIP were preferably engulfed by granulocytes in comparison to polymeric NP. The size of the NP was also found to be a determinant factor as both positively and negatively charged large NP were internalized to a similar extent by granulocytes in-vivo, whereas SN-NP were not internalized by granulocytes. Lymphocytes preferably engulfed large negatively charged formulations, LN-LIP and LN-NP.

\section{Conclusions}

Systemically administrated nanocarriers are engulfed by three leukocytes types, monocytes, neutrophils, and B-cells, but not T cells. Ex-vivo experiments failed to accurately distinguish between cells type engaged in the uptake of liposomes and polymeric NP. SN-NP $(84.8 \pm 25.6 \mathrm{~nm},-14.6 \pm 1.7 \mathrm{mv})$ exhibited the highest selectivity towards monocytes, and were internalized almost exclusively by these cells. Granulocytes preferentially internalized LN-LIP $(130 \pm 32.7 \mathrm{~nm},-25.2 \pm 6.3$ $\mathrm{mv})$, and lymphocytes preferably internalized large 
negative liposomes, and polymeric NP $(133.3 \pm 40.1 \mathrm{~nm}$, $-39.9 \pm 7.5 \mathrm{~nm})$.

In conclusion, while designing a formulation targeted to monocytes, it is important to take under consideration its distribution into other leukocytes. Optimizing the physicochemical characteristics can achieve higher selectivity, improved efficacy and reduce side effect. In order to examine the performance of a new formulation for monocytes, an in-vivo model should be utilized, since meaningful conclusions can be drawn from in-vivo experiments only.

\section{Acknowledgment}

This work was supported in part by Biorest, Israel.

\section{References}

1. Farokhzad OC and Langer R. Nanomedicine: developing smarter therapeutic and diagnostic modalities. Adv Drug Deliv Rev 2006; 58: 1456-9. doi:10.1016/j.addr.2006.09.0 $\underline{11}$

2. Zhang L, Gu FX, Chan JM, Wang AZ, Langer RS and Farokhzad OC. Nanoparticles in medicine: therapeutic applications and developments. Clin Pharmacol Ther 2008; 83: 761-9. doi:10.1038/sj.clpt.6100400

3. Li SD and Huang L. Pharmacokinetics and biodistribution of nanoparticles. Mol Pharm 2008; 5: 496-504. doi:10.102 $1 / \mathrm{mp} 800049 \mathrm{w}$

4. Ishida $\mathrm{T}$, Harashima $\mathrm{H}$ and Kiwada $\mathrm{H}$. Liposome clearance. Biosci Rep 2002; 22: 197-224. doi:10.1023/A:102013452 $\underline{1778}$

5. Moghimi SM, Hunter AC and Murray JC. Nanomedicine: current status and future prospects. FASEB J 2005; 19: 311-30. doi:10.1096/fj.04-2747rev

6. Owens DE, 3rd and Peppas NA. Opsonization, biodistribution, and pharmacokinetics of polymeric nanoparticles. Int J Pharm 2006; 307: 93-102. doi:10.1016/j.ijpharm.2005.10.0 $\underline{10}$

7. Alexis F, Pridgen E, Molnar LK and Farokhzad OC. Factors affecting the clearance and biodistribution of polymeric nanoparticles. Mol Pharm 2008; 5: 505-15. doi:10.1021/m p800051m

8. Moghimi SM, Hunter AC and Murray JC. Long-circulating and target-specific nanoparticles: theory to practice. Pharmacol Rev 2001; 53: 283-318.

9. Afergan E, Epstein H, Dahan R, Koroukhov N, Rohekar K, Danenberg HD and Golomb G. Delivery of serotonin to the brain by monocytes following phagocytosis of liposomes. $\mathrm{J}$ Control Release 2008; 132: 84-90.

10. Bakker-Woudenberg IA, Storm G and Woodle MC. Liposomes in the treatment of infections. J Drug Target 1994; 2: 363-71. doi:10.3109/10611869408996811

11. Danenberg HD, Fishbein I, Gao J, Monkkonen J, Reich R, Gati I, Moerman E and Golomb G. Macrophage depletion by clodronate-containing liposomes reduces neointimal formation after balloon injury in rats and rabbits. Circulation 2002; 106: 599-605.
12. van Rooijen $\mathrm{N}$ and Sanders A. Liposome mediated depletion of macrophages:mechanism of action, preparation of liposomes and applications. J Immunol Methods 1994; 174: 83-93. doi:10.1016/0022-1759(94)90012-4

13. van Rooijen $\mathrm{N}$ and van Kesteren-Hendrikx E. Clodronate liposomes: perspectives in research and therapeutics. J Liposome Res 2002; 12: 81-94. doi:10.1081/LPR-120004780

14. Cohen-Sela E, Dangoor D, Epstein H, Gati I, Danenberg HD, Golomb G and Gao J. Nanospheres of a bisphosphonate attenuate intimal hyperplasia. J Nanosci Nanotechnol 2006; 6: 3226-34.

15. Cohen-Sela E, Rosenzweig O, Gao J, Epstein H, Gati I, Reich R, Danenberg HD and Golomb G. Alendronateloaded nanoparticles deplete monocytes and attenuate restenosis. J Controlled Rel 2006; 113: 23-30.

16. Danenberg HD, Fishbein I, Epstein H, Waltenberger J, Moerman E, Monkkonen J, Gao J, Gathi I, Reich R and Golomb G. Systemic depletion of macrophages by liposomal bisphosphonates reduces neointimal formation following balloon-injury in the rat carotid artery. J Cardiovasc Pharmacol 2003; 42: 671-9.

17. Danenberg HD, Golomb G, Groothuis A, Gao J, Epstein H, Swaminathan RV, Seifert P and Edelman ER. Liposomal alendronate inhibits systemic innate immunity and reduces in-stent neointimal hyperplasia in rabbits. Circulation 2003; 108: 2798-804.

18. Markovsky E, Koroukhov N and Golomb G. Additive-free albumin nanoparticles of alendronate for attenuating inflammation through monocyte inhibition. Nanomed 2007; 2: 545-53.

19. Haber E, Danenberg HD, Koroukhov N, Ron-El R, Golomb $\mathrm{G}$ and Schachter M. Peritoneal macrophage depletion by liposomal bisphosphonate attenuates endometriosis in the rat model. Hum Reprod 2009; 24: 398-407.

20. Cohen-Sela E, Chorny M, Koroukhov N, Danenberg HD and Golomb G. A new double emulsion solvent diffusion technique for encapsulating hydrophilic molecules in PLGA nanoparticles. J Control Release 2009; 133: 90-5.

21. Fessi H, Devissaguet JP, Puisieux F and Thies C. U.S. patent 1992; 5: 528.

22. Chorny M, Polyak B, Alferiev IS, Walsh K, Friedman G and Levy RJ. Magnetically driven plasmid DNA delivery with biodegradable polymeric nanoparticles. FASEB J 2007; 21: 2510-9. doi:10.1096/fj.06-8070com

23. Szoka F, Jr. and Papahadjopoulos D. Comparative properties and methods of preparation of lipid vesicles (liposomes). Annu Rev Biophys Bioeng 1980; 9: 467508. doi:10.1146/annurev.bb.09.060180.002343

24. Epstein H, Berger V, Levi I, Eisenberg G, Koroukhov N, Gao J and Golomb G. Nanosuspensions of alendronate with gallium or gadolinium attenuate neointimal hyperplasia in rats. J Controlled Rel 2007; 117: 322-32.

25. Bucher DJ, Kharitonenkov IG, Zakomirdin JA, Grigoriev VB, Klimenko SM and Davis JF. Incorporation of influenza virus M-protein into liposomes. J Virol 1980; 36: 586-90.

26. Leo E, Contado C, Bortolotti F, Pavan B, Scatturin A, Tosi G, Manfredini S, Angusti A and Dalpiaz A. Nanoparticle formulation may affect the stabilization of an antiischemic 
nanobe.org

prodrug. Int J Pharm 2006; 307: 103-13. doi:10.1016/j.ijph arm.2005.09.031

27. Gibaud S, Demoy M, Andreux JP, Weingarten C, Gouritin $\mathrm{B}$ and Couvreur P. Cells involved in the capture of nanoparticles in hematopoietic organs. J Pharm Sci 1996; 85: 944-50. doi:10.1021/js960032d

28. Zambaux MF, Faivre-Fiorina B, Bonneau F, Marchal S, Merlin JL, Dellacherie E, Labrude P and Vigneron C. Involvement of neutrophilic granulocytes in the uptake of biodegradable non-stealth and stealth nanoparticles in guinea pig. Biomaterials 2000; 21: 975-80. doi:10.1016/S01 42-9612(99)00233-1

29. Ke Y and Kapp JA. Exogenous antigens gain access to the major histocompatibility complex class I processing pathway in B cells by receptor-mediated uptake. J Exp Med 1996; 184: 1179-84. doi:10.1084/jem.184.3.1179

30. Blumenthal R, Weinstein JN, Sharrow SO and Henkart P. Liposome--lymphocyte interaction: saturable sites for transfer and intracellular release of liposome contents. Proc Natl Acad Sci U S A 1977; 74: 5603-7. doi:10.1073/pnas.74.1 $\underline{2.5603}$

31. Leroux JC, Gravel P, Balant L, Volet B, Anner BM, Allemann E, Doelker E and Gurny R. Internalization of poly(D,L-lactic acid) nanoparticles by isolated human leukocytes and analysis of plasma proteins adsorbed onto the particles. J Biomed Mater Res 1994; 28: 47181. doi:10.1002/jbm.820280410

32. Shen H, Ackerman AL, Cody V, Giodini A, Hinson ER, Cresswell P, Edelson RL, Saltzman WM and Hanlon DJ. Enhanced and prolonged cross-presentation following endosomal escape of exogenous antigens encapsulated in biodegradable nanoparticles. Immunology 2006; 117: 7888. doi:10.1111/j.1365-2567.2005.02268.x

33. Zhang JS, Liu F and Huang L. Implications of pharmacokinetic behavior of lipoplex for its inflammatory toxicity. Adv Drug Deliv Rev 2005; 57: 689-98. doi:10.1016/j.add r.2004.12.004

34. Sunderkotter C, Nikolic T, Dillon MJ, Van Rooijen N, Stehling M, Drevets DA and Leenen PJ. Subpopulations of mouse blood monocytes differ in maturation stage and inflammatory response. J Immunol 2004; 172: 4410-7.

35. Swirski FK, Nahrendorf M, Etzrodt M, Wildgruber M, Cortez-Retamozo V, Panizzi P, Figueiredo JL, Kohler RH, Chudnovskiy A, Waterman P, Aikawa E, Mempel TR, Libby $\mathrm{P}$, Weissleder $\mathrm{R}$ and Pittet MJ. Identification of splenic reservoir monocytes and their deployment to inflammatory sites. Science 2009; 325: 612-6. doi:10.1126/science.11 $\underline{75202}$

Received 2 May, 2010; accepted 6 June, 2010; published online 26 June, 2010.

Copyright: (C) 2010 E. Cohen-Sela et al. This is an open access article distributed under the terms of the Creative Commons Attribution License, which permits unrestricted use, distribution, and reproduction in any medium, provided the original author and source are credited. 